\title{
Every group is isomorphic to a group of permutations
}

\author{
Open Mathematics Collaboration*† \\ March 22, 2021
}

\begin{abstract}
CAYLEY'S THEOREM, the SYMMETRIC GROUP THEOREM, and their underlying definitions are presented in this white paper (knowledge base = http://omkb.org).
\end{abstract}

keywords: Cayley's theorem, symmetric group, isomorphism, abstract algebra, knowledge base

The most updated version of this white paper is available at https://osf .io/63pmy/download

*All authors with their affiliations appear at the end of this white paper.

†Corresponding author: mplobo@uft.edu.br|Open Mathematics Collaboration 


\section{Cayley's Theorem}

1.

$$
(G, \star) \cong\left(P, \circ_{\alpha}\right)
$$

$(G, \star):=$ group

$\left(P, \circ_{\alpha}\right):=$ permutation group

$\cong$ isomorphism

$\star, \circ_{\alpha}:=$ binary operations

$\alpha: P \rightarrow P \quad$ (permutation $:=$ bijective function)

$\circ_{\alpha}:=$ composition of permutations

$[1,2]$

\section{Symmetric Group on $A$}

2.

$$
(S(A), \circ)
$$

$S(A)=\{f \mid f: A \cong A\}:=$ set of permutations of $A$

$A:=$ nonempty set

$f: A \cong A \quad$ (bijective function)

$\circ:=$ operation of composition

$[1,2]$ 


\section{Prerequisites}

3. Ordered pair

$$
(a, b)=\{\{a\},\{a, b\}\}
$$

$a:=$ first coordinate

$b:=$ second coordinate

$[1,3]$

4. Cartesian product

$$
A \times B=\{(a, b) \mid a \in A, b \in B\}
$$

$A, B:=$ sets

$A \times B:=$ Cartesian product

$(a, b):=$ ordered pair

$[3]$

5. Binary operation

$$
\star: S \times S \rightarrow S
$$

$S:=$ set

$S \times S:=$ Cartesian product

[1]

6. Group

$$
(G, \star)
$$

(a) Associativity: $\forall x, y, z \in G,(x \star y) \star z=x \star(y \star z)$

(b) Identity: $\exists e \in G: \forall x \in G, e \star x=x \star e=x$

(c) Inverse: $\forall x \in G \exists y \in G: x \star y=y \star x=e$ 
$G:=$ set

$\star:=$ binary operation

$[1]$

7. Relation from $A$ to $B$

$$
R \subseteq A \times B
$$

$R, A, B:=$ sets

$x \in A, y \in B, \quad x R y \equiv(x, y) \in R$

$[3]$

8. Function from $A$ to $B$

$$
\begin{gathered}
f: A \rightarrow B \\
\forall a \in A \exists ! b \in B((a, b) \in f)
\end{gathered}
$$

$f, A, B:=$ sets

$(a, b):=$ ordered pair

$[3]$

9. One-to-one function (injection) $f: A \rightarrow B$

$$
\neg \exists a_{1} \in A \exists a_{2} \in A\left(f\left(a_{1}\right)=f\left(a_{2} \wedge a_{1} \neq a_{2}\right)\right)
$$

$[3]$

10. Onto function (surjection) $f: A \rightarrow B$

$$
\forall b \in B \exists a \in A(f(a)=b)
$$

$[3]$ 
11. Bijective function := one-to-one + onto

$[3]$

12. Permutation of $A:=$ bijection from $A$ to itself

$[1,2]$

13. Homomorphism

$$
f^{h}
$$

$$
\begin{aligned}
& \text { (a) } f^{h}: G \rightarrow H \\
& \forall x, y \in G: f^{h}(x * y)=f^{h}(x) \circ f^{h}(y) \\
& f^{h}:=\text { function } \\
& G, H:=\text { sets } \\
& *, \circ:=\text { binary operations } \\
& (G, *),(H, \circ):=\text { groups } \\
& {[1,4,5]}
\end{aligned}
$$

14. Isomorphism $:=$ bijective homomorphism $[1,4,5]$

\section{Open Invitation}

Review, add content, and co-author this white white paper [6,7]. Join the Open Mathematics Collaboration.

Send your contribution to mplobo@uft.edu.br. 


\section{Open Science}

The latex file for this white paper together with other supplementary files are available in [8].

\section{Ethical conduct of research}

This original work was pre-registered under the OSF Preprints [9], please cite it accordingly [10]. This will ensure that researches are conducted with integrity and intellectual honesty at all times and by all means.

\section{Acknowledgements}

+ Center for Open Science https://cos.io

+ Open Science Framework https://osf.io

\section{References}

[1] Warner, Steve. Abstract Algebra for Beginners. GET 800, 2018. https://books.google.com/books?id=UFleyAEACAAJ

[2] Dixon, John D., and Brian Mortimer. Permutation groups. Vol. 163. Springer Science \& Business Media, 1996. https://books.google.com/books?vid=1SPjBwAAQBAJ

[3] Velleman, Daniel J. How to prove it: A structured approach. Cambridge University Press, 2019. https://books . google.com/books?vid=ISBN0521861241 
[4] Dummit, David Steven, and Richard M. Foote. Abstract Algebra. Vol. 3. Hoboken: Wiley, 2004.

https://books . google.com/books?id=znzJygAACAAJ

[5] Rotman, Joseph J. A first course in abstract algebra. Pearson College Division, 2000.

https://books.google.com/books?id=ctEZAQAAIAAJ

[6] Lobo, Matheus P. "Microarticles." OSF Preprints, 28 Oct. 2019. https://doi.org/10.31219/osf.io/ejrct

[7] Lobo, Matheus P. "Simple Guidelines for Authors: Open Journal of Mathematics and Physics." OSF Preprints, 15 Nov. 2019.

https://doi.org/10.31219/osf .io/fk836

[8] Lobo, Matheus P. "Open Journal of Mathematics and Physics (OJMP)." OSF, 21 Apr. 2020.

https://doi.org/10.17605/osf.io/6hzyp

https://osf.io/6hzyp/files

[9] COS. Open Science Framework. https://osf .io

[10] Lobo, Matheus P. "Every Group Is Isomorphic to a Group of Permutations." OSF Preprints, 22 Mar. 2021.

https://doi.org/10.31219/osf .io/63pmy

\section{The Open Mathematics Collaboration}

Matheus Pereira Lobo (lead author, mplobo@uft.edu.br) $)^{1,2}$ https://orcid.org/0000-0003-4554-1372

${ }^{1}$ Federal University of Tocantins (Brazil)

${ }^{2}$ Universidade Aberta (UAb, Portugal) 